\title{
Vertical Signal Flow and Oscillations in a Three-Layer Model of the Cortex
}

\author{
URSULA FUENTES, RAPHAEL RITZ* ${ }^{*}$ WULFRAM GERSTNER, \\ AND J. LEO VAN HEMMEN \\ lwh@Physik.TU-Muenchen.de \\ Phyxik-Department der Tll München. D-85747 Garching bei München. Germany
}

Received August 16, 1994. Revised October 10, 1995.

Action Editor: Charles Gray

\begin{abstract}
A model of vertical signal flow across a layered cortical structure is presented and analyzed. Neurons communicate through spikes, which evoke an excitatory or inhibitory postsynaptic potential (spike response model). The layers incorporate two anatomical features - dendritic and axonal arborization patterns and distance-dependent time delays. The vertical signal flow through the network is discussed for various stimulus conditions using two different, but typical, axonal arborization patterns. We find stationary as well as oscillatory response, but the oscillatory response may be restricted to a single layer. Confronted with conflicting stimuli the network separates the patterns through phase-shifted oscillations. We also discuss two hypothetical animals, to be called "cat" and "mouse." These have different axonal arborizations, which give rise to a different oscillatory response (if any) of the various layers.
\end{abstract}

Keywords: neural modeling, laminar structure, collective oscillations

\section{Introduction}

A section of the mammalian cortex reveals a clear laminar structure in the vertical direction, whereas the connectivity is isotropic in the tangential plane (see, e.g., Kandel et al., 1991). The layered structure is reflected in the vertical range of the dendritic and axonal arborization of pyramidal cells. That is, the afferent and efferent connectivity is layer-dependent (Braitenberg and Schüz, 1991; Felleman and van Essen, 1991). Ascending projections tend to terminate in layer IV (granular layer), whereas feedback connections from higher areas usually project to infragranular and supragranular layers, avoiding layer IV (Nakajima et al., 1988; Cauller and Connors, 1994). These findings suggest a complicated pattern of vertical signal flow.

In this paper, the question of signal flow in the cortex is related to the problem of existence (or nonexistence) of collective $30-50 \mathrm{~Hz}$ oscillations, which have been observed in cat and monkey visual cortex (Eckhorn et al., 1988; Gray and Singer, 1989; Gray et al., 1989;

* present address: Computational Neurobiology Laboratory, The Salk Institute for Biological Studies, POB 85800, San Diego, CA 92186-5800, USA
Kreiter and Singer, 1992). Coherent oscillations with zero phase-lag have been detected within one column between different layers (Eckhorn et al., 1988), cells exhibiting oscillatory responses being located primarily in supra- and infragranular layers, rarely in layer IV (Gray et al., 1990). This synchronized activity could be a mechanism for feature linking, acting as a temporal label and thus solving the problem of global object perception (von der Malsburg, 1981; von der Malsburg and Schneider, 1986; Eckhorn et al., 1988; for a critical discussion see Ghose and Freeman, 1992).

Here we develop a model of the layered cortical structure in order to study the vertical signal flow in a small slab of cortex (e.g., a hypercolumn of $1 \mathrm{~mm}$ diameter). We focus on the question whether collective oscillations (if they occur at all) are restricted to a single layer or whether they are spread out vertically over the full hypercolumn. The model described here is an extension of the spike response model, SRM (Gerstner et al., 1993ab; Ritz et al., 1994), which includes many biological details such as time delays and realistic EPSP and IPSP shapes.

Our model of a layered structure is similar to the models of Krone et al., (1986), Thomas et al., (1991), 
and Patton et al., (1992). In contrast to these models, which are based on a coding by the mean firing rate, the SRM describes single spikes of single neurons. In addition, our model shows some associative structure similar to the Hopfield model (Hopfield, 1982).

\section{Definition of the Model}

The underlying network model is the spike response model (SRM), which has been introduced before (Gerstner et al., 1993ab; Ritz et al., 1994). After a brief summary of the main features, the model is extended so as to deal with different cortical layers.

\subsection{Spike Response Model}

We consider a network of $N$ fully connected model neurons that is able to store $q$ palterns $(1 \leq \mu \leq q)$ using a Hebbian learning rule. After learning, the synaptic efficacics $J_{i j}$ of signal transmission from neuron $j$ to neuron $i$ are (van Hemmen et al., 1990)

$$
J_{i j}=\frac{2}{N\left(1-a^{2}\right)} \sum_{\mu=1}^{q} \xi_{i}^{\mu}\left(\xi_{j}^{\mu}-a\right)
$$

where the $\left\{\xi_{i}^{\mu}= \pm 1 \mid 1 \leq i \leq N\right\}$ are random patterns and $a$ denotes the mean activity of the patterns $a \equiv\left\langle\xi_{i}^{\mu}\right\rangle=\frac{1}{q N} \sum_{\mu=1}^{q} \sum_{i=1}^{N} \xi_{i}^{\mu}$. Each of these Hebbian neurons is also connected to an inhibitory partner neuron. By this extreme form of locality we mimic the local inhibition caused by, for example, spiny stellate cells as observed in the mammalian cortex. Correspondingly, the Hebbian neurons represent the cortical pyramidal cells, which exhibit long-range interaction. Spikes are described by a formal variable $S_{i}(t) \in\{0,1\}$ (where $S_{i}(t)=1$ denotes a single spike) and take a typical spike width of $1 \mathrm{~ms}$ as the basic time step of our model. We use parallel (that is, simultaneous) updating, the dynamics of neuron $i$ being defined by the probability of firing during one time step, given a membrane potential $h_{i}$,

$$
\begin{aligned}
& \operatorname{Pr}\left[S_{i}(t+1)=+1 \mid h_{i}(t)\right]= \\
& \frac{1}{2}\left[1+\tanh \left(\beta\left\{h_{i}[S(t)]-\vartheta\right\}\right)\right],
\end{aligned}
$$

where we have introduced a noise-parameter $\beta$ and a threshold $\vartheta$.

In the SRM, the membrane potential $h_{i}(t)$ consists of three components

$$
h_{i}(t)=h_{i}^{\mathrm{syn}}(t)+h_{i}^{\mathrm{rcf}}(t)+h_{i}^{\mathrm{ext}}(t),
$$

where $h_{i}^{\text {ext }}$ describes an external stimulus, $h_{i}^{\text {syn }}$ is the input of all other neurons, and $h_{i}^{\text {ref }}$ models the absolute refractory period of duration $\tau_{\text {ref. }}$. If we take

$$
h_{i}^{\text {ref }}= \begin{cases}-R & \text { for } t_{F} \leq t \leq t_{F}+\tau_{\text {ref }} \\ 0 & \text { otherwise }\end{cases}
$$

and $R \gg 1$, then firing is prevented during a time $\tau_{\text {ref }}$ after emission of a spike at $t=t_{F}$. Here we choose ${ }^{\cdot} \tau_{\text {ref }}=1 \mathrm{~ms}$. The synaptic component $h_{i}^{\text {syn }}$ is the sum of the inputs from all other neurons, both Hebbian and inhibitory,

$$
h_{i}^{\mathrm{syn}}=h_{i}^{\mathrm{hebb}}+h_{i}^{\mathrm{inh}} .
$$

If a Hebbian neuron fires $\left(S_{i}=+1\right)$, the inhibitory partner neuron is also excited. After a delay $\Delta_{i}^{i n h}$, the inhibition by the partner neuron is fed back to the Hebbian neuron. Using a memory kernel $\eta(\tau)$, we have

$$
h_{i}^{\mathrm{inh}}(t)=\sum_{\tau=0}^{\tau_{\max }} \eta(\tau) S_{i}\left(t-\tau-\Delta_{i}^{\mathrm{inh}}\right) .
$$

The IPSP $\eta(\tau)$ starts with a steep increase to a saturation value $\eta_{\max }$ and decays exponentially with a time constant $\tau_{\eta}=6 \mathrm{~ms}$ afterwards - that is,

$$
\eta(\tau)=-\eta_{\max } \exp \left(-\tau / \tau_{\eta}\right)
$$

The upper bound $\tau_{\max }$ in (6) is a soft maximum and adjusted in such a way that summation is stopped after the first nonvanishing term, a simple means to include saturation effects. The delay $\Delta_{i}^{\mathrm{inh}}$ is a random variable sampled with uniform distribution from $\Delta_{\min }^{\mathrm{inh}} \leq \Delta_{i}^{\mathrm{inh}} \leq \Delta_{\max }^{\mathrm{inh}}$. Similarly, connections between Hebbian neurons have a transmission delay $\Delta_{i}$, which is taken to be a stochastic variable in a range $\Delta_{\min } \leq \Delta_{i} \leq \Delta_{\max }$. Spikes induce a postsynaptic potential

$$
h_{i}^{\text {hebb }}=\sum_{j=1}^{N} J_{i j} \sum_{\tau=0}^{\infty} \epsilon(\tau) S_{j}\left(t-\tau-\Delta_{i}\right)
$$


with a time course $\epsilon(t)$, here taken to be an alpha function

$$
\epsilon(t)=\alpha \frac{t}{\tau_{\epsilon}} \exp \left(-\frac{t}{\tau_{\epsilon}}\right)
$$

normalized such that $\sum_{t} \epsilon(t)=1$, and the amplitude given by the synaptic efficacies $J_{i j}$.

Note that all important features of neuronal signal transmission are summarized in the delays and the time course of the response functions $\eta(\tau)$ and $\epsilon(\tau)$.

As we have shown previously (Gerstner et al., 1993a), three different states can evolve, depending on the timing of the EPSP $\epsilon(\tau)$ in relation to the IPSP $\eta(\tau)$ : weakly locked oscillatory, stationary, or perfectly locked oscillatory activity.

\subsection{Layered Cortical Structure}

In the model presented here, we assume three different layers, each containing $N$ pairs of neurons as described in the previous subsection. The upper layer (layer 1) models the cortical supragranular layers, whereas the lower layer (layer 3 ) represents the infragranular layers. Layer 2 corresponds to the cortical input layer IV. The reduction of six cortical layers to three model layers is suggested by common interareal connections. A uniform distribution of $3 N$ neurons to three layers is not far from reality, as one can conclude from experimental data on cell densities and thickness of layers (Creutzfeldt, 1983).

We assume full connectivity of the Hebbian neurons in each layer, leading to an isotropic tangential structure, consistent with the known homogeneous wiring structure in the tangential plane of a small cortical slab. The vertical structure of, for example, a hypercolumn is introduced into the SRM by two new features:

- a layer-dependent vertical range of axonal and dendritic arborization and

- distance-dependent dendritic and axonal delays instead of a stochastic distribution; the latter is quite popular in theoretical work.

Our main aim here is to study the effect of these features on the collective activity of the neurons in different layers. Also, the question appears which of the two approaches is more effective - the anatomical specification of the layers or the temporal delays between them.
First, let us consider the dendritic and axonal arborization, which is layer-dependent (Lund et al., 1979; Creutzfeldt, 1983; Gilbert and Wiesel, 1983; Valverde, 1984; Braitenberg, 1986; Krone et al., 1986; Burkhalter, 1989; Douglas and Martin, 1991; Anderson et al., 1993; Gilbert, 1993). Specifically, we assume the following dendritic arborization (see Fig. 1A). For a neuron in layer $l$ the dendrites ramify in layer $l$ and in all layers above layer $l$, in agreement with typical cortical dendritic arborization as observed in different mammals.

Regarding the axonal arborization, experimental results vary between different mammals. We will therefore take the axonal branching pattern as variable and study its effects on the collective activity of neurons in different layers.

Given the axonal and dendritic branching pattern, we have to define a connection scheme between neurons located in different layers. Since cortical axons and dendrites are found to be densely covered with synapses (Braitenberg, 1986), one can assume that there is a synaptic connection whenever a dendrite of one ncuron touches an axon of another ncuron. We go even further and make the following assumption. Given neuron $i$ in layer $l(1 \leq l \leq 3)$ and neuron $j$ in layer $k(1 \leq k \leq 3)$, there is a connection from neuron $j$ to neuron $i$ in each layer which is passed or reached by both axons of neurons in layer $k$ and dendrites of neurons in layer $l$ (see Fig. 1B).

In passing we note that it depends on the chosen axonal branching pattern whether the $3 N$ neurons are fully connected or not, if the dendritic arborization is given as described above.

We now turn to the temporal aspects of a layered cortical structure. In our model, we have chosen a time delay of $1 \mathrm{~ms}$ per model layer for both dendritic and axonal signal transmission, which seems to be a realistic assumption (Patton et al., 1992). This leads to a delay

$$
\Delta_{l}(m, k)=|k-m|+|m-l| \quad \begin{aligned}
& 1 \leq l, k \leq 3 \\
& 1 \leq m \leq l
\end{aligned}
$$

for signal transmission from neurons in layer $k$ to those in layer $l$ with a synaptic connection in layer $m$ (see Fig. 1B). The dendritic arborization has been taken into account in the upper bound $l$ for the values of $m$ (that is, for the location of synaptic connections). We then have $\Delta_{l}(m, k) \in\{0, \ldots, l+1\}$, and $\Delta_{l}(m, k) \leq 4 \mathrm{~ms}$. This means that if axonal arborization leads to a fully 
A Spread of the dendrites

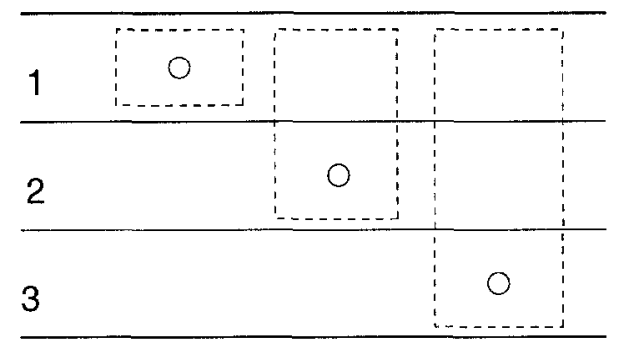

C Spread of the axons for $A^{C}$

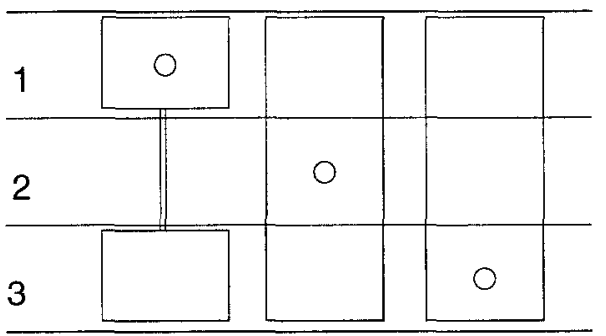

B Pathways from $\mathrm{j}$ to $\mathrm{i}$

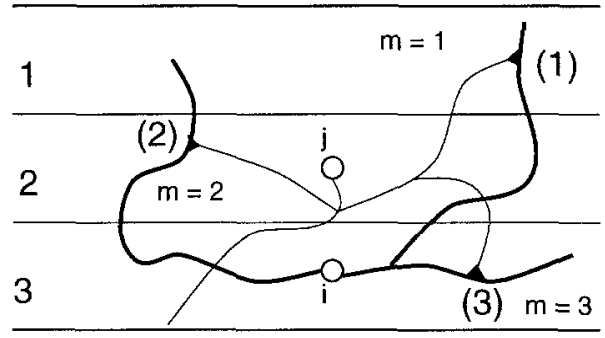

D Spread of the axons for $A^{m}$

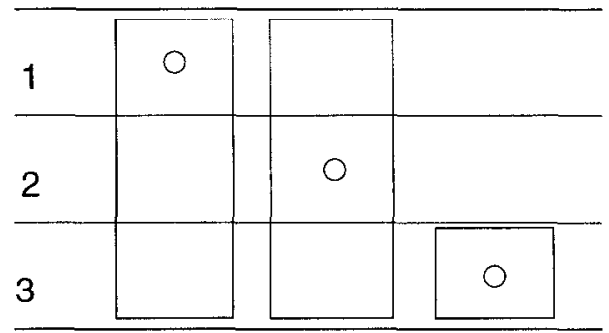

Fig. 1. Structure of the layered network: Threc layers representing the supragranular (I-III), input (IV), and infragranular (V, VI) layers of the cortex are interconnected according to the above scheme. A The dendrites of a neuron in layer $l(l=1,2,3$ from left to right) ramify in layer $l$ and in all layers above layer $l$. B Different pathways between two neurons may exist and result in different delays (as illustrated for neuron $j$ of layer 2 with neuron $i$ in layer 3 ). One pathway (1) via a synapse in layer 1 leads according to (10) to a delay $\Delta_{3 i}^{12}=3 \mathrm{~ms}$, while for the pathways (2) and (3) a delay $\Delta_{3 i}^{22}=\Delta_{3 i}^{32}=1 \mathrm{~ms}$ arises. C, D Axonal branching structure for $A^{\mathrm{c}}$ and $A^{\mathrm{m}}$, respectively.

connected network, we end up in the regime of weakly locked oscillatory activity, because the delay times are comparatively short (Gerstner et al., 1993a).

The layered network learns a set of $q$ random patterns comprising all three layers. Specifically, a pattern $\mu$ is defined as a set of independent, identically distributed, random variables $\xi_{l i}^{\mu}$, with $1 \leq \mu \leq q$, $1 \leq l \leq 3$, and $1 \leq i \leq N$, which assume the values $\xi_{l i}^{\mu}=\perp 1$ with probability $(1 \perp a) / 2$, where $a$ is, as before, the mean activity of the learned patterns. Learning these patterns yields the synaptic efficacies (van Hemmen et al., 1990)

$$
\begin{aligned}
J_{l i, k j}= & \frac{2}{3 N\left(1-a^{2}\right)} \sum_{\mu=1}^{q} \xi_{l i}^{\mu}\left(\xi_{k j}^{\mu}-a\right) \\
& 1 \leq l, k \leq 3 \\
& 1 \leq i, j \leq N
\end{aligned}
$$

independent of the location $m$ of the synapse. Compared to (1) we have slightly changed the notation and the normalization.
The synaptic efficacies determine the amplitude of the contribution of all other Hebbian neurons to the postsynaptic potential of neuron $i$ in layer $l$

$$
\begin{aligned}
h_{l i}^{\text {hebb }}=\sum_{k=1}^{3} \sum_{m=1}^{l} A_{k m} \sum_{j=1}^{N} J_{l i, k j} & \\
& \sum_{\tau=0}^{\infty} \epsilon(\tau) S_{k j}\left(t-\tau-\Delta_{l i}^{m k}\right),
\end{aligned}
$$

with the response function $\epsilon(\tau)$ as in (9). The matrix $A_{k m}$ represents the axonal branching pattern (see Fig. 1C, D for two examples). That is, we have $A_{k m}=0$ if neurons in layer $k$ do not send axonal collaterals into layer $m$, and $A_{k m}=1$ if they do. The dendritic arborization has been taken into account through the upper bound $l$ for the summation over the synaptic locations $m$, in agreement with the assumption that dendritic trees of neurons in layer $l$ extend to all layers above layer $l$, including layer $l$ itself.

As a measure for the correlation hetween the state of the neurons in one layer and the corresponding part 
of the learned pattern $\mu$ we introduce the overlaps

$$
\begin{aligned}
m_{\mu}^{k}(t)= & \frac{2}{N\left(1-a^{2}\right)} \sum_{j=1}^{N}\left(\xi_{k j}^{\mu}-a\right) S_{k j}(t) \\
& 1 \leq k \leq 3 \\
& 1 \leq \mu \leq q .
\end{aligned}
$$

The overlap takes a maximum value, if all neurons of layer $k$ that belong to the foreground of pattern $\mu$ $\left(\xi_{k j}^{\mu}=1\right)$ fire synchronously during one time step while all the background neurons $\left(\xi_{k j}^{\mu}=-1\right)$ stay quiescent. It vanishes, if firing of foreground as well as background neurons occurs stochastically and $N$ is large $(N \rightarrow \infty)$.

With the above definitions (10) through (12), the synaptic contribution to the postsynaptic potential of neuron $i$ in layer $l$ is

$$
\begin{aligned}
h_{l i}^{\mathrm{syn}}= & \frac{1}{3} \sum_{k=1}^{3} \sum_{m=1}^{l} A_{k m} \sum_{\mu=1}^{q} \xi_{l i}^{\mu} \\
& \sum_{\tau=0}^{\infty} \epsilon(\tau) m_{\mu}^{k}\left(t-\tau-\Delta_{l i}^{m k}\right) \\
+ & \sum_{\tau=0}^{\tau_{\max }} \eta(\tau) S_{l i}\left(t-\tau-\Delta_{l i}^{\mathrm{inh} h}\right) .
\end{aligned}
$$

Simulations of the extended SRM, eqs. (2) through (13), are discussed now.

\section{Simulation Results}

In this section, we present the results of simulations with a network of $N=2,000$ neuron pairs in each of the three model layers, which was trained on $q=5$ patterns of low activity $(a=-0.8)$. In passing we note that a higher loading would not change the network performance as long as $q$ is of the order of $N$ but the computational effort would increase substantially, so we refrained from doing so. The inhibitory delay times have been chosen from the interval [ $2 \mathrm{~ms}, 5 \mathrm{~ms}$ ] and the time constant of the response function (9) is taken to be $\tau_{\epsilon}=2 \mathrm{~ms}$. During 1,000 time-steps the network is updated simultaneously according to (2) with finite noise $(\beta=15)$ and a threshold $\vartheta=0.14$. We start with a randomly chosen state of activity $a=-0.8$. After $200 \mathrm{~ms}$ a week external signal $h_{l i}^{\text {ext }}=\gamma\left(\xi_{l i}^{\mu}+1\right) / 2$, with $\gamma=0.22$, is switched on in one or more layers $l$. This external signal is switched off after another $600 \mathrm{~ms}$. First, we stimulate only layer $l=2$, which corresponds to the input-layer IV of the cortex. In a second step, we also study the system under an input in layers 1 and 3, which represent the supra- and infragranular layers where feedback signals from higher areas arrive.

\subsection{Feedforward Input}

In this subsection, we study the network activity in all layers for different axonal branching patterns $A_{k m}$ in the case of input into layer $l=2$ only, that is $h_{l i}^{\text {ext }}=\delta_{l 2} \gamma\left(\xi_{l i}^{\mu}+1\right) / 2$. Biologically, this can be interpreted as a stimulation of input-layer IV, such as in the primary visual cortex by signals coming from the thalamus.

It can be extracted from neuroanatomical data about the visual cortex of cats (Lund et al,, 1979; Gilbert and Wiesel, 1983; Douglas and Martin, 1991; Gilbert, 1993), monkeys (Tigges and Tigges, 1982; Valverde, 1984; Anderson et al., 1993), and men (Creutzfeldt, 1983) that pyramidal cells in supragranular layers send thcir axons vertically downwards and leave the cortex to terminate in other cortical areas or in subcortical structures. On their way through the cortex they send out collaterals in regular intervals, but generally avoiding layer IV. The axons of pyramidal cells located in other layers also leave the cortex having crossed the layers below them, where they send out collaterals. In addition, there are collaterals which reach the upper layers (see Fig. 1C).

These neuroanatomical observations can be incorporated schematically in our model using the following matrix to describe the axonal branching pattern

$$
A=\left(\begin{array}{lll}
1 & 0 & 1 \\
1 & 1 & 1 \\
1 & 1 & 1
\end{array}\right) \equiv A^{\mathrm{c}} .
$$

Here, the superscript $c$ should be considered as a label for a branching pattern that is inspired by some anatomical findings in the cat, but we do not claim to model the cortex of a cat in any detail, especially since there may be no connections from layer IV to layer $V$ in real cats (Gilbert and Wiesel, 1983; Gilbert, 1993). In short, c refers to a hypothetical animal. With this form of the malrix $A_{k m}$, all neurons of our model network send their axons to all layers except neurons in layer $k=1$, which do not send axons into layer $m=2\left(A_{12}=0\right)$. Nevertheless, the network as a whole is fully connected, 
Table 1. Delays $\Delta_{l}(m, k)$ according to (10) for signal transmission from neurons in layer $k$ to neurons in layer $l$ through synapses in layer $m$, for $A^{\mathrm{c}}$ as the axonal branching pattern. The dendritic arborization is restricted to $m \leq l$.

\begin{tabular}{|c|c|c|c|c|c|c|c|}
\hline \multicolumn{8}{|c|}{$\Delta_{l}(m, k)$ for $A^{\mathrm{c}}$} \\
\hline \multirow[b]{2}{*}{ k } & \multirow{2}{*}{$\begin{array}{l}1 \\
\mathrm{~m}\end{array}$} & \multirow{2}{*}{$\begin{array}{l}1 \\
1\end{array}$} & \multicolumn{2}{|c|}{2} & \multicolumn{3}{|c|}{3} \\
\hline & & & 1 & 2 & 1 & 2 & 3 \\
\hline 1 & & 0 & 1 & - & 2 & - & 2 \\
\hline 2 & & 1 & 2 & 0 & 3 & 1 & 1 \\
\hline 3 & & 2 & 3 & 1 & 4 & 2 & 0 \\
\hline
\end{tabular}

as one can see in Table 1, where we listed those time delays that arise with (10) and (15), taking into account the given dendritic branching. Each delay time $\Delta_{l}(m, k)$ represents existing connections from neurons in layer $k$ to neurons in layer $l$ over synapses in layer $m$.

The result of the simulation for $A^{\mathrm{c}}$ as defined in (15) is shown in Fig. 2. Note the collective oscillation $(25-30 \mathrm{~Hz})$ in layers 1 and 3, whereas the overlap with the stimulated pattern is nearly stationary in input layer 2. This result is in accordance with some experimental results on collective oscillations in visual cortex from cats (Eckhorn et al., 1988; Gray et al., 1990), where almost no oscillations could be found in the input layer. Since the depicted overlap is obtained during a single run, this ensemble averaged quantity clearly reflects every synchronized activity as soon as a substantial amount of neurons takes part in it. So, by just looking at the plots one gets a hint from the time structure whether collective activity is present and to what extend. This is already enough for the questions we are interested in here. Concerning the drawbacks of such a straightforward approach and how to reveal arbitrary phase relations the reader is referred to Gerstner et al., (1993b).

The axonal branching pattern of mice and rats (Krone et al., 1986) seems to be different from the one described for, for example, cats and monkeys in that pyramidal cells only send axonal collaterals into their own layer and the layers below. Cells in layer IV, however, have additional recurrent axons that reach the upper layers (see Fig. 1D), whereas rats show an even higher extent of connectivity (Burkhalter, 1989). These principles of axonal branching can be modeled by means of the following branching matrix:

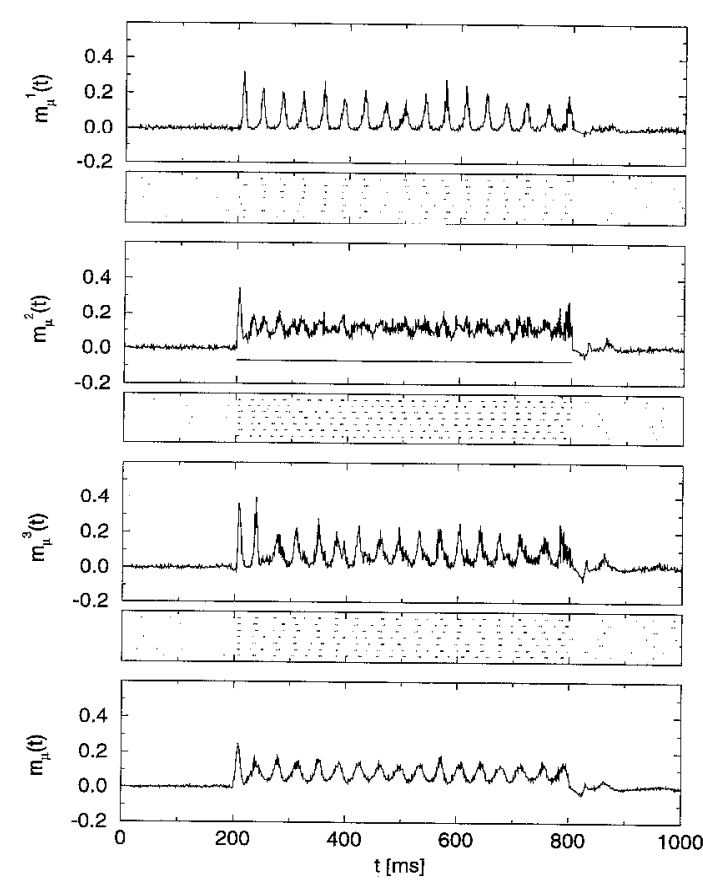

Fig. 2. Simulation results for $A^{\mathrm{c}}$ : The overlaps $m_{\mu}^{k}$ with a stimulated pattern $\mu$ are shown as a function of time for the three different layers $k, 1 \leq k \leq 3$, together with a spike raster of seven representative neurons of pattern $\mu$ in each layer. Finally, the total overlap with pattern $\mu, m_{\mu}$, is plotted. The pattern is presented from $t=200 \mathrm{~ms}$ to $800 \mathrm{~ms}$ but only to neurons of layer 2 , the input layer, as indicated by the horizontal bar underneath the overlap. Note the collective oscillation $(25-30 \mathrm{~Hz})$ in layers 1 and 3 , which receive no stimulus, while in the input layer 2 the overlap is almost stationary.

Table 2. Delays $\Delta_{l}(m, k)$ according to (10) for signal transmission from neurons in layer $k$ to neurons in layer $l$ through synapses in layer $m$, using $A^{\mathrm{m}}$ as axonal branching matrix and the given dendritic arborization $m \leq l$.

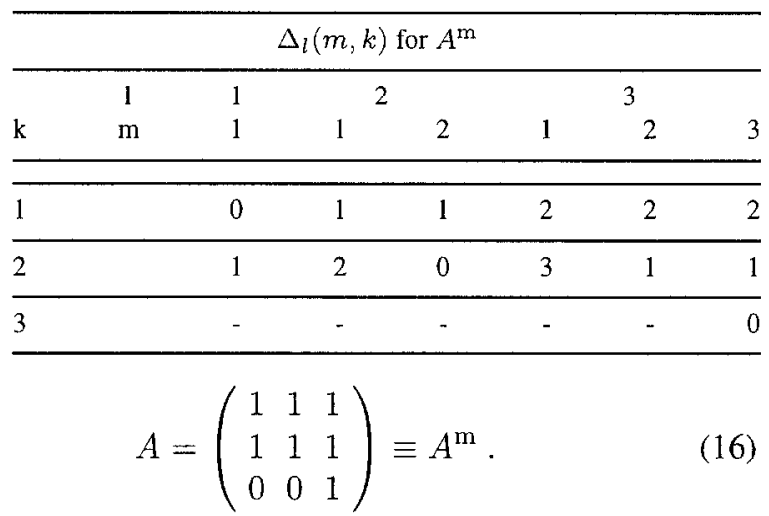

With this matrix, the network is no longer fully connected hecause there is no connection from neurons in layer $k=3$ to neurons in layers $l=1$ and $l=2$ 


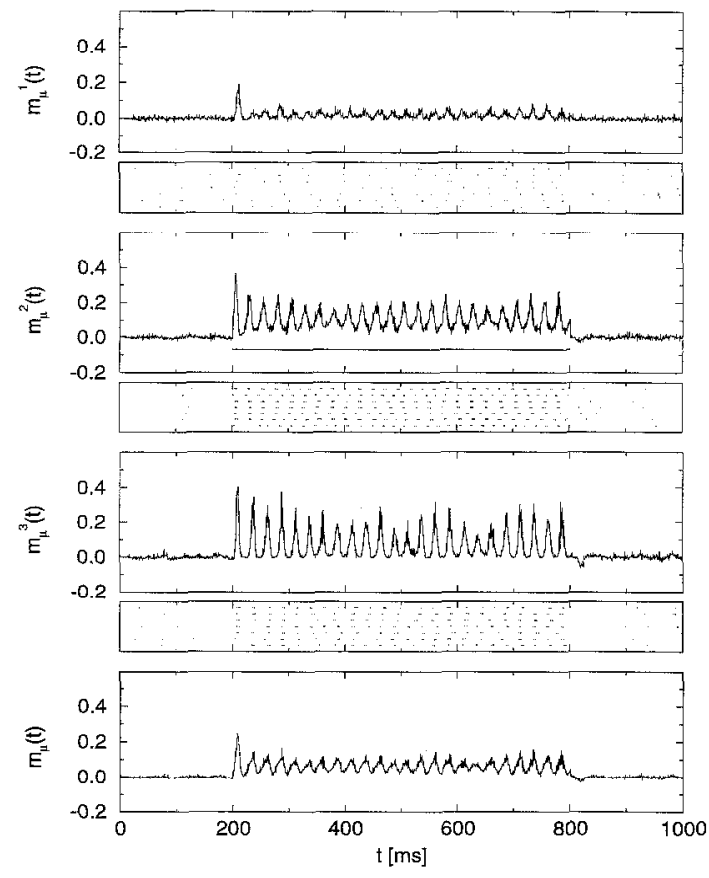

Fig. 3. Simulation results for $A^{\mathrm{m}}$ : The plot is organized as before (see Fig. 2). Due to the change in the arborization pattern, a stimulation of the input layer 2 now induces a collective oscillation in all three layers hut with a higher frequency $(40 \mathrm{~Hz}$ ) as compared to the scenario $A^{\mathrm{c}}$. In layer 1 , however, the activity is only slightly above the spontaneous activity, as can clearly be seen in the spike raster, because there is only one pathway feeding input into this layer (see Table 2).

( $A_{31}=A_{32}-0$ and $m \leq l$ ). Thus the network has less intracolumnar feedback than with (15). The superscript $m$ now refers to an other hypothctical animal (for convenience the reader may think of a mouse but we concentrate here only on a different connectivity scheme).

The simulation of the network dynamics using (16) shows an collective oscillatory activity $(40 \mathrm{~Hz})$ in layers 2 and 3 (scc Fig. 3). In layer 1, however, the amplitude of the response to the stimulus in layer 2 exceeds only slightly the spontaneous activity because neurons in layer $l=1$ receive signals only from neurons in their own layer and from layer $k=2$, with only one synaptic connection per neuron. On the other hand, the amplitude of the oscillation in layer 3 is larger than that in layer 2, although only layer 2 receives an input signal. The reason for this effect is the dendritic branching structure, which leads to seven synaptic connections per neuron for layer $l=3$ in contrast to four connections per neuron for layer $l=2$ (see Table 2).
The dynamical evolution in layer 2 is almost independent of the other layers because it receives signals from only layers 1 and 2 and the activity of layer 1 can be neglected. The appearance of a collective oscillation of $40 \mathrm{~Hz}$ in layers 2 and 3 is easy to understand. Because of the short delay times, the excitation of a neuron in layer 2 due to the spikes of other neurons in the same layer arrives when the neuron is still shunted by local inhibition. After the IPSP has declined, the external input is again able to fire some neurons, which due to the short delay times can drag some other neurons along. This also explains why the oscillation stops when the stimulus is switched off (see scenario I in Gerstner et al., 1993a). Layer 3 receives a strong input from layer 2 and thus displays the same dynamics as layer 2 . Note that there is a small phase lag in the response of layer 3 relative to the input layer.

But why does the axonal branching matrix $A^{\mathrm{C}}$ lead to oscillations in layers 1 and 3 , and why is the frequency smaller than for $A^{\mathrm{m}}$ ? To answer these questions, we study the effect of additional feedback via the parameters $A_{31}$ and $A_{32}$.

If we take

$$
A=\left(\begin{array}{lll}
1 & 1 & 1 \\
1 & 1 & 1 \\
0 & 1 & 1
\end{array}\right) \equiv A^{\text {trans }}
$$

as an axonal branching pattern instead of (16), we have an additional feedback. In contrast to (16) there now is a connection from layer $l=3$ to layer $l=2$.

We have simulated a network with the connectivity matrix $A^{\text {trans }}$, driven by an external input into layer 2 . In this layer we find a stationary overlap with the stimu lus pattern, while layer 3 exhibits collective oscillatory activity, but with a longer oscillation period as compared to the scenarios before (see Fig. 4). The breakdown of the collective oscillation in layer 2 found for $A^{\mathrm{m}}$ can be explained by the phase lag in the response in layer 3 , which is now fed back into layer 2 . The oscillation period in layer 3 is longer than for $A^{\mathrm{m}}$ because this layer receives a stationary input from layer 2 (activity in layer 1 being negligible) with an amplitude less than $\gamma=0.22$ (for $m_{\mu}^{2}<0.22$, see Fig. 4), which means that it takes longer for the IPSP to decline far enough so as to allow the input to layer 3 to be strong enough to stimulate the neurons again.

If we turn on the feedback parameter $A_{31}$ (that is choosing the matrix $A_{k m}-1$ for all $k, m$ as axonal branching matrix $A^{\text {full }}$ ), we arrive at a fully connected 


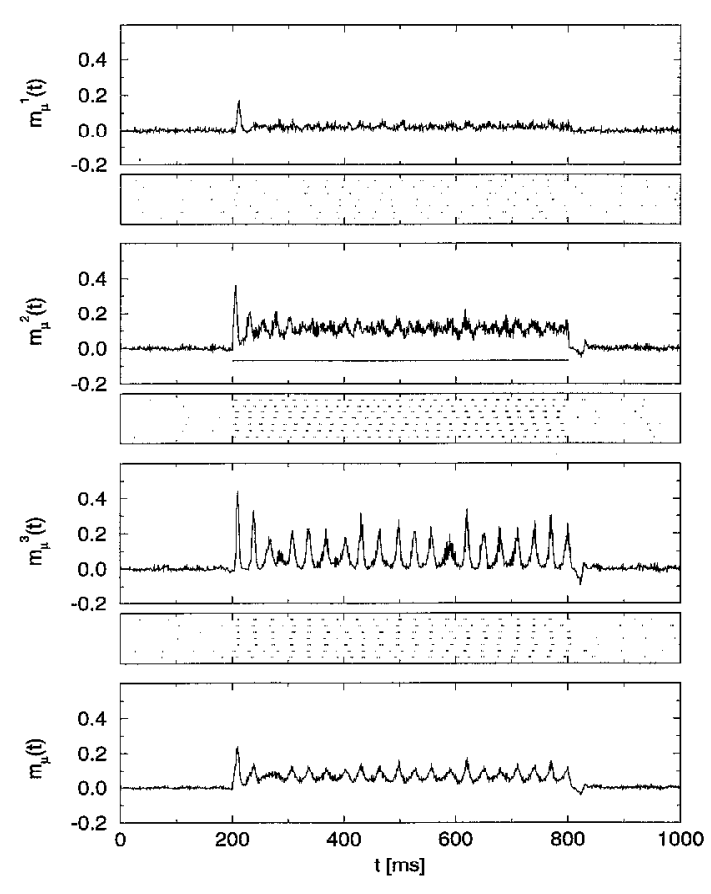

Fig. 4. Simulation results for $A^{\text {trans }}$ : The only structural difference as compared to the scenario $A^{\mathrm{m}}$ before (Fig. 3) is that we now allow feedback from layer 3 to the input layer $2\left(A_{32}=1\right)$, resulting in two changes in the netwnrk's response. First, the oscillation in layer 2 is suppressed. Second, the frequency is lowered to $33 \mathrm{~Hz}$. We refer to Section 3.1 for an explanation of these effects.

network. The only difference compared to $A^{\mathrm{c}}$ is that the connection from layer 1 to layer 2 is stronger. So it is not surprising that simulation results are quite similar, except that layer 2 exhibits a slightly oscillatory overlap with the stimulated pattern because of the stronger oscillatory input from layer 1 (see Fig. 5).

\subsection{Feedback from Higher Cortical Areas}

In this subsection we present simulations with an external input into the model layers 1 and 3, which correspond to the supra- and infragranular layers, which receive feedback signals from higher cortical areas (anatomical data showing this may be found in Cauller and Connors, 1994, for rats and in Nakajima et al., 1988 , for cats).

For $A^{\mathrm{c}}$ the simulation yields a collective oscillatory activity in layer 2 and practically stationary overlap with the stimulated pattern in input layers 1 and 3, whereas for $A^{\mathrm{m}}$ we have a stationary overlap with the stimulated pattern in layer 1 and synchronous oscilla-

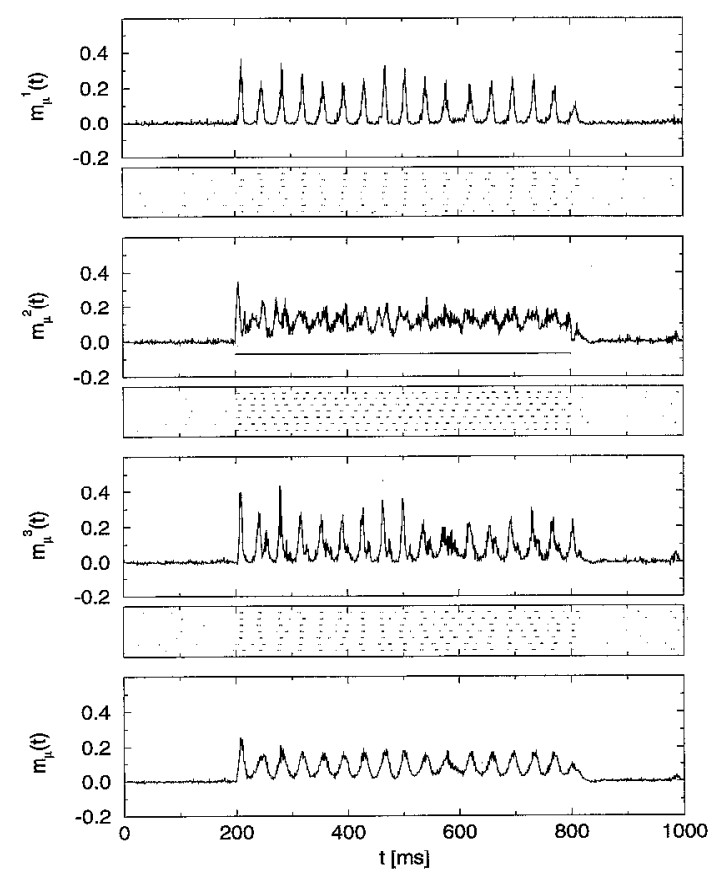

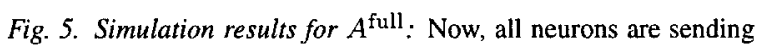
axons to all layers $\left(A_{k m}=1\right.$ for all $k$ and $m$ ). As a result, the response to a stimulus is oscillatory in the layers 1 and 3 with a frequency of $28 \mathrm{~Hz}$ while it is almost stationary in the input layer where the stimulus is fed into (see Fig. 2, where we had $A_{12}=0$ ).

tory activity in layer 3 , the response of layer 2 being negligible (see Fig. 6).

More interesting in this context is the following question: What happens if the feedback from higher cortical areas corresponds to a different pattern than the one evoked by the stimulus? This can be interpreted as a conflict between the internal expectation and the outside world. In our model, this can be described by an stimulus $h^{\text {ext }}$, which is different in layer 2 (external input) than in layers 1 and 3 (internal feedback stimulus). Thus we have

$$
\begin{aligned}
& h_{1 i}^{\text {ext }}=\gamma^{\mathrm{fb}}\left(\xi_{1 i}^{\nu}+1\right) / 2 \\
& h_{2 i}^{\text {ext }}=\gamma^{\mathrm{ext}}\left(\xi_{2 i}^{\mu}+1\right) / 2 \\
& h_{3 i}^{\text {ext }}=\gamma^{\mathrm{fb}}\left(\xi_{3 i}^{\nu}+1\right) / 2 .
\end{aligned} \quad \text { with } \mu \neq \nu
$$

The results of a simulation with this stimulus $\left(\gamma^{\text {ext }}=\right.$ $0.22, \gamma^{\mathrm{fh}}=0.18$ ) are shown in Fig. 7 for the axonal branching structure $A^{\mathrm{m}}$. In layer 1 , we find an oscillatory response $(30 \mathrm{~Hz})$ of the overlap, which is mainly correlated with pattern $\nu$, the pattern supported by the cortical feedback. Similarly in layer 2 the overlap with pattern $\mu$, the external stimulus, dominates. In layer 

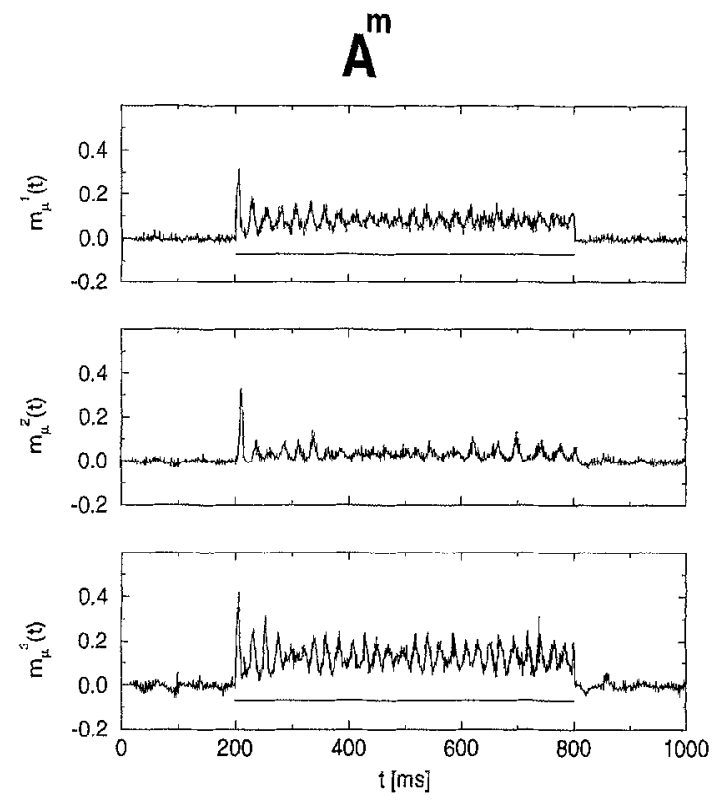
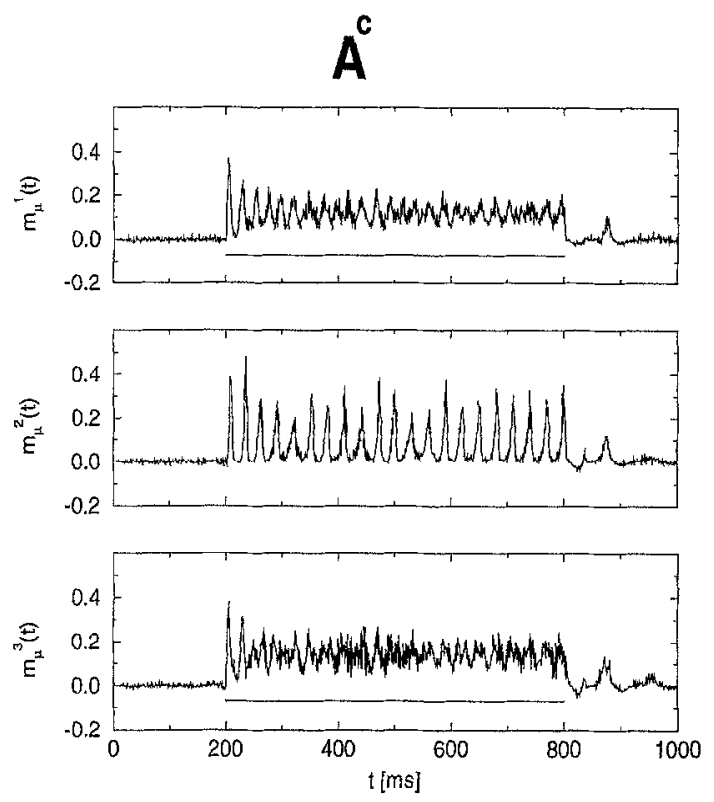

Fig. 6. Feedback from higher cortical areas: Simulation results for the stimulation of pattern $\mu$ in layers 1 and $3(\gamma=0.22)$ as indicated by the horizontal lines for the axonal branching patterns $A^{\mathrm{m}}$ (left) and $A^{\mathrm{c}}$ (right). We plot the overlaps $m_{\mu}^{k}$ with the stimulus pattern $\mu$ as a function of time for all three layers separately. While for $A^{\mathrm{m}}$ the network tends to an oscillatory response in the stimulated layers ( 1 and 3 ), this is different for $A^{\mathrm{c}}$. Here a pronounced oscillation is found in layer 2 .

3 , however, we get a different response. Both patterns are activated in a collective oscillation $(30 \mathrm{~Hz})$ of similar strength but phase-shifted (see Fig. 7). Thus, in layer 3 both patterns are present. They do not appear, however, in a mixture state but are separated through a phase shift of the respective activity.

On the other hand, with the axonal branching pattern $A^{\mathrm{c}}$ we find a separated activation of both patterns throughout the whole network (see Fig. 8). This is not surprising because due to the higher connectivity for $A^{\mathrm{c}}$ as compared to $A^{\mathrm{m}}$ the external signal as well as the feedback input is mediated strongly to all neurons - as we have seen in Section 3.1 for the case of a single input into layer 2 .

\subsection{Simulations Without Distance-Dependent De- lays}

Until now we have modeled a layered cortical structure based on two features, a layer-dependent vertical range of dendritic and axonal arborization on the one hand and distance-dependent time delays on the other hand. In order to find out if the anatomical laminar structure as described by layer-dependent arborization alone leads to the described layer-dependent collective activity or whether the temporal aspect of layered structure is essential, the same simulations as described in the above subsections were performed with delay-times $\Delta_{i i}$ chosen stochastically out of an interval $\left[0 ; \Delta^{\max }\right]$. For $\Delta^{\max } \leq 3$ the results are the same as with distance-dependent delays according to (10). This is in agreement with results obtained previously from homogeneous networks (Gerstrer et al., 1993a; Ritz et al., 1994). There, it has been shown that, as long as the excitatory transmission delays are shorter than the inhibitory ones, the network is in the weak locking regimc, i.e., stimulus induced collective oscillations are possible. For a more detailed discussion of results regarding the existence and delay dependence of collective oscillations in the SRM, see Gerstner et al. (1993a) and Ritz et al. (1994). This suggests that the specific distribution of time delays that results from the anatomical structure of dendritic and axonal arborization is not essential in our model here. What is important is the connectivity resulting from the vertical arborization. 

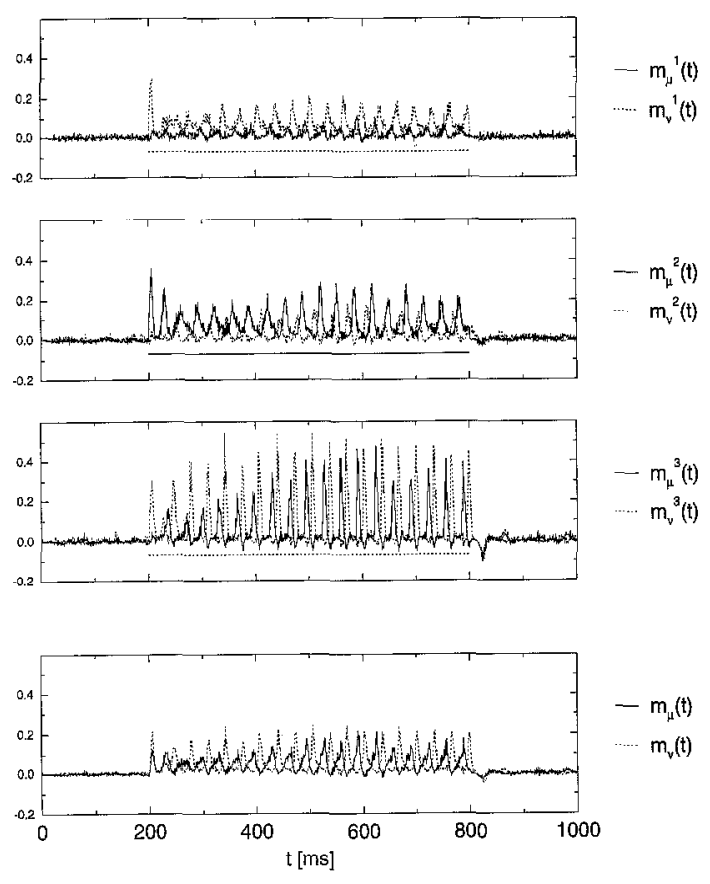

Fig. 7. Conflicting stimuli for $A^{\mathrm{m}}$ : Simulation results for the stimulation of pattern $\mu$ in layer 2 ( $\gamma^{\text {ext }}=0.22$; solid lines) and pattern $\nu$ in layers 1 and $3\left(\gamma^{\mathrm{fb}}=0.18\right.$; dotted lines) for the axonal branching pattern $A^{\mathrm{m}}$. In layer 1 we have a strong overlap with pattern $\nu$, while in layer 2 pattern $\mu$ dominates. In layer 3 , however, both patterns are activated but phase-shifted with respect to each other. This is interpreted as a separation of the two patterns.

\section{Discussion}

Taking advantage of the spike response model, SRM (Gerstner et al., 1993ab; Ritz et al., 1994) we have introduced a layered structure that includes two major elements of cortical organization - typical arborization patterns for dendrites and axons in the vertical direction and, second, realistic distance-dependent dendritic and axonal delays. Local inhibition and synaptic responses are taken into account by response kernels for the EPSP and IPSP as in the SRM.

The general branching pattern is based on the idea that neurons from layer $l(1 \leq l \leq 3)$ get input from all layers above $l$ (including $l$ itself) according to available anatomical data (Lund et al., 1979; Creutzfeldt, 1983; Gilbert and Wiesel, 1983; Valverde, 1984; Braitenberg, 1986; Krone et al,, 1986; Burkhalter, 1989; Douglas and Martin, 1991; Anderson et al., 1993; Gilbert, 1993). Specifically, we have considered two different, anatomically inspired, axonal branching patterns, $A^{\mathrm{c}}$ and $A^{\mathrm{m}}$, under various stimulus conditions. Stimulation of model layer 2 (corresponding to layer IV of the cortex) describes external say, sensory, input whereas layers 1 and 3 are (if identified with the supra- and infragranular layers of the cortex) the terminals for feedback signals from higher cortical areas.

Our results can be summarized as follows. Stimulation of layers 1 and 3 has a different effect on the network than stimulation of layer 2 ; This result is independent of the branching pattern. The characteristic response for $A^{\mathrm{m}}$ is an oscillation in the frequency range of 40 to $45 \mathrm{~Hz}$ in layer 3 and in the layer that is directly stimulated by external or cortical input. For $A^{\mathrm{c}}$, however, we typically get no oscillation in the directly stimulated layers, but only in the others. The oscillation period ranges from 30 to $35 \mathrm{~Hz}$.

The layered network is also capable of pattern segmentation when confronted with conflicting stimuli from the external and feedback inputs (as in Wang et al., 1990, and Ritz et al., 1994, for a superposition of several stimuli in the external input). Both patterns are activated coherently but phase-shifted, in the case of $A^{\mathrm{c}}$ throughout the whole network, for $A^{\mathrm{m}}$ only in layer 3. Thus the systems response to a conflict between reality and expectation consists in an ambivalent reaction: both patterns remain active but separated. This is true for almost uncorrelated patterns as is the case here where we have random patterns in the low loading, low activity limit. We did not study the case of corrclated patterns since then the learning rule (1) breaks down, but it would be interesting to look at different models, where correlated patterns could be handled - such as using the unlearning algorithm (Hopfield et al., 1983; van Hemmen et al., 1990; Wimbauer et al., 1994).

The observed layered response seems to be due mainly to the vertical arborization structure and not primarily to the distance-dependent delays, as can be concluded from the different responses for $A^{\mathrm{c}}$ and $A^{\mathrm{m}}$ to stimulation of layer 2 only. Additional feedback from layer 3 to layers 2 and 1 removed the differences as shown in the branching patterns $A^{\text {trans }}$ and $A^{\text {full }}$.

A characteristic feature of the present three-layer network is increasing activation from layer 1 to layer 3 , due to the dendritic arborization. This is based on the idea that dendrites of neurons in the lower layers are longer than those of neurons on top of the cortex and seems to be a realistic assumption regarding the mean cortical anatomy (Krone et al., 1986; von Seelen et al.,1987). As a consequence of this feature the activity of the lowest layer 3 is strong enough to excite the upper layer 1 even if there is only a weak signal 

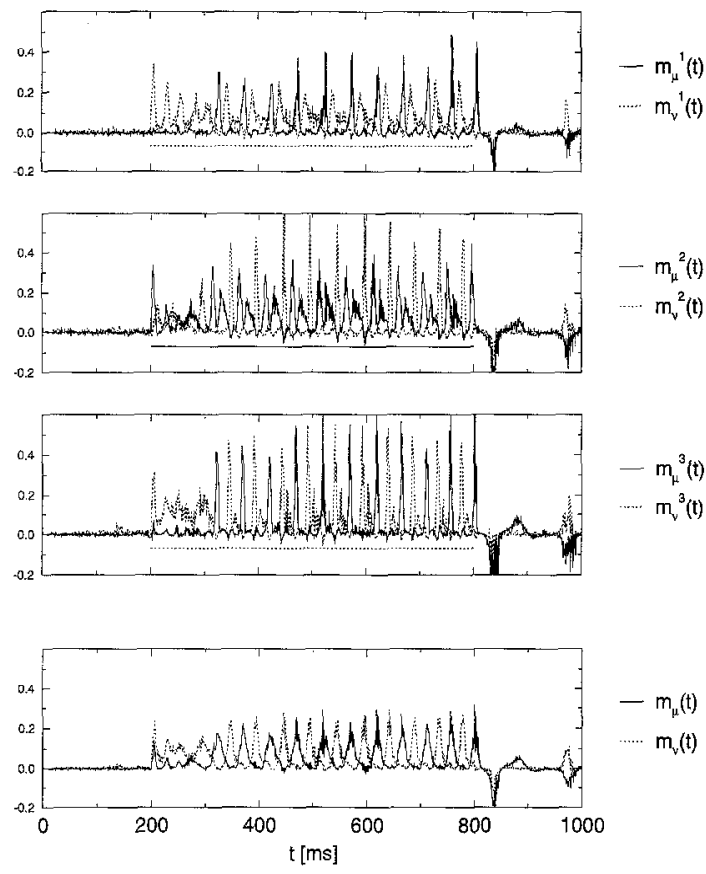

Fig. 8. Conflicting stimuli for $A^{\mathrm{c}}$ : Taking the same stimulus as before (Fig. 7) but the branching structure $A^{\mathrm{C}}$, we get pattern segmentation throughout the whole network (stimulus for layer 2: pattern $\mu, \gamma^{\text {ext }}=0.22$, solid lines; stimulus for layers 1 and 3: pattern $\nu, \gamma^{\mathrm{tb}}=0.18$, dashed lines).

to layer 2 provided there are enough connections from layer 3 to layer 1 as with the branching pattern $A^{c}$. The direct connections from layer 2 to layer 1 do not suffice as was shown for the branching pattern $A^{\mathrm{m}}$.

Regarding our connectivity scheme wc follow the model of Krone et al., (1986), but we reduce it to three layers and neglect all latcral structurc. Thus, our nctwork is structurally more transparent, but - we hope still capable of producing biologically relevant results. The major difference to Krone et al., (1986) is in the dynamics. Whilc Kronc ct al., (1986) usc a lincar dynamics for the mean firing rate we have implemented a nonlinear, noisy thrcshold dynamics producing single spikes together with realistic delays and synaptic response kerncls. In contrast to Thomas et al., (1991) and Patton et al., (1992), who use an extremely detailed model of the structural as well as the dynamical properties of single neurons we have concentrated on the study of structural principlcs manifcsted in the organization of the cortex. A side advantage is (though not shown here) that the dynamics can be treated analytically as well.
Finally, the question arises: what are the documented effects good for? As long as there are only speculative assumptions about the functional role of collective oscillations (if there is any), this answer can't be given here relying only on a model study. Of course, it is tempting to speculate that these phenomena are reflecting parts of the neuronal correlate of visual awareness, attention, or other higher brain functions (Koch and Crick, 1994; Singer, 1994; Crick and Koch, 1995). In that case, our findings would suggest that there are fundamental differences in higher cortical functions between different species due to the wiring of their cortical tissue even at such an early stage as the primary visual cortex. Whether one of these interpretations could result in a testable prediction regarding the performance of these two species in a psychophysical paradigm remains an outstanding question in the context of one of the greatest mysteries science can ever deal with.

\section{Acknowledgements}

This work has been supported by the Deutsche Forschungsgemeinschaft (grant number: He 1729/2-1 and 2). UF gratefully acknowledges support from the Studienstiftung des Deutschen Volkes.

\section{References}

Anderson JC, Martin KAC, and Whitteridge D (1993) Form, function, and intracortical projections of neurons in the striate cortex of the monkey Macacus nemestrinus. Cerebral Cortex 3:412-420.

Braitenberg V (1986) Two views of the cerebral cortex. In: Palm G, and Aertsen A, eds. Brain Theory. Springer, Berlin, Heidelberg, New York. pp. 81-96.

Braitenberg V, and Schiiz A (1991) Anatomy of the cortex. Springer, Berlin, Heidelherg, New York. ch. 28.

Burkhalter A (1989) Intrinsic connections of rat primary visual cortex: Laminar organization of axonal projections. J. Comp. Neurol. 279:171-186.

Cauller LJ, and Connors BW (1994) Synaptic physiology of horizontal afferents to layer I in slices of rat SI neocortex. J. Neurosci. 14:751-762.

Creutzfeldt OD (1983) Cortex Cerebri. Springer Berlin, Heidelberg, New York.

Crick F, and Koch C (1995) Are we aware of neural activity in primary visual cortex? Nature 375:121-123.

Douglas RJ, and Martin KAC (1991) A functional microcircuit for cat visual cortex. J. Physiol. (London) 440:735-769.

Eckhorn R, Bauer R, Jordan W, Brosch M, Kruse W, Munk M, and Reitboeck HJ (1988) Coherent oscillations: A mechanism of feature linking in the visual cortex? Biol. Cybern. 60:121-130. 
Felleman DJ, and van Essen DC (1991) Distributed hierarchical processing in the primate cerebral cortex. Cerebral Cortex $1: 1-47$.

Fuentes U (1993) Einfluß der Schicht- und Arealstruktur auf die Informationsverarbeitung im Cortex. Diplomarbeit, Technische Universität München.

Gerstner W, Ritz R, and van Hemmen JL (1993a) A biologically motivared and analytically soluble model of collective oscillations in the cortex: I. Theory of weak locking. Biol. Cybern. 68:363-374.

Gerstner W, Ritz R, and van Hemmen JL (1993b) Why spikes? Hebbian learning and retrieval of time-resolved excitation patterns. Biol. Cybern. 69:503-515.

Ghose GM, and Freeman RD (1992) Oscillatory discharge in the visual system: Does it have a functional role? $J$. Neurophysiol. 68:1558-1574.

Gilbert CD (1993) Circuitry, architecture, and functional dynamics of visual cortex. Cerebral Cortex 3:373-386.

Gilbert CD, and Wiesel TN (1983) Clustered intrinsic connections in cat visual cortex. J. Neurosci. 3:1116-1133.

Gray CM, Engel AK, König P, and Singer W (1990) Stimulusdependent neuronal oscillations in cat visual cortex: Receptive field properties and feature dependence. Eur. J. Neurosci. 2 (1990):607-619.

Gray CM, König P, Engel AK, and Singer W (1989) Oscillatory responses in cat visual cortex exhibit inter-columnar synchronization which reflects global stimulus properties. Nature 338:334-337.

Gray CM, and Singer W (1989) Stimulus-specific neuronal oscillations in orientation columns of cat visual cortex. Proc. Natl. Acad. Sci. USA 86:1698-1702.

Hopfield JJ (1982) Neural networks and physical systems with emergent collective computational abilities. Proc. Natl. Acad. Sci. USA 79:2554-2558.

Hopfield Jy, Feinstein DI, and Palmer RG (1983) Unlearning has a stabilizing effect in collective memories. Nature 304:158-159.

Kandel ER, Schwartz JH, and Jessell TM (1991) Principles of neuroscience (3rd ed.). Prentice-Hall, London.

Koch C, and Crick F (1994) Some further ideas regarding the neuronal basis of awareness. In: Koch C, and Davies JL, cds. Largescale neuronal theories of the brain. MIT Press, Cambridge, MA. pp. 93-109.

Kreiter AK, and Singer W (1992) Oscillatory neuronal responses in the visual cortex of the awake macaque monkey. Eur. J. Neurosci. 4:369-375.

Krone G, Mallot H, Palm G, and Schüz A (1986) Spatiotemporal receptive fields: a dynamical model derived from cortical architectonics. Proc. R. Soc. Lond., Ser. B 226:421-444.

Lund JS, Henry GH, MacQueen CL, and Harvey AR (1979) Anatomical organization of the primary visual cortex (Area 17) of the cat.
A comparison with Area 17 of the macaque monkey. I. Comp. Neur. 184:599-618.

Nakajima S, Komatsu Y, and Toyama K (1988) Synaptic action of layer I fibers on cells in cat striate cortex. Brain Res. 457:176-180.

Patton P, Thomas E, and Wyatt RE (1992) A computational model of vertical signal propagation in the primary visual cortex. Biol. Cybern. 68:43-52.

Ritz R, Gerstner W, Fuentes U, and van Hemmen JL (1994) A biologically motivated and analytically soluble model of collective oscillations in the cortex: II. Application to binding and pattern segmentation. Biol. Cybern. 71:349-358.

Singer W (1994) Putative functions of temporal correlations in nencortical processing. In: Koch $\mathrm{C}$, and Davies $\mathrm{JL}$, eds. Large-scale neuronal theories of the brain. MIT Press, Cambridge, MA. pp. 201-237.

Thomas E, Patton P, and Wyatt RE (1991) A computational model of the vertical anatomical organization of primary visual cortex. Biol. Cybern. 65:189-202.

Tigges J, and Tigges M (1982) Principles of axonal collateralization of laminae II-III pyramids in Area 17 of squirrel monkey: A quantitative Golgi study. Neuroscience Letters 29:99-104.

Valverde $F(1984)$ The organizing principles of the primary visual cortex in the monkey. In: Peters A, and Jones EG, eds. Cerebral Cortex (Vol. 3). Plenum Press, New York, London.

van Hemmen JL, Gerstner W, Herz AVM, Kühn R, Sulzer B, and Vaas M (1990) Encoding and decoding of patterns which are correlated in space and time. In: Dorffner $\mathrm{G}$, ed. Konnektionismus in Artificial Intelligence und Kognitionsforschung. Springer, Berlin, Heidelberg, New York. pp. 153-162.

van Hemmen JL, loffe LB, Kühn R, and Vaas M (1990) Increasing the efficiency of a ncural network through unlcaming. Physica $A$ 163:386-392.

von der Malsburg C (1981) The correlation theory of brain function. Internal Report 81-2, MPI für Biophysikalische Chemie, Göttingen. Reprinted in: Domany E, van Hemmen JL, and Schulten K, eds. (1994) Models of Neural Networks II. Springer, New York. ch. 2.

von der Malsburg C, and Schneider W (1986) A neural cocktail-party processor. Biol. Cybern. 54:29-40.

von Seelen W, Mallot HA, and Giannakopoulos F (1987) Characteristics of neural systems in the visual cortex. Biol. Cybern. 56:37-49.

Wang D, Buhmann J, and von der Malsburg C (1990) Pattern segmentation in associative memory. Neural Comp. 2:94-106.

Wimbauer S, Klemmer N, and van Hemmen JL (1994) Universality of unlearning. Neural Networks 7:261-270. 